\title{
CÓMO INTEGRAR EL ARTE Y LA LITERATURA A LAS CLASES DE ESPAÑOL
}

\section{RESUMEN}

La utilización de la literatura y del arte puede convertirse en una herramienta de contacto con la cultura y la historia del idioma meta, además de facilitar el desarrollo y la integración de los contenidos básicos encontrados en los manuales didácticos. En este taller traemos sugerencias prácticas de usos del arte y de la literatura en clases de español como lengua extranjera. De esta manera buscamos ayudar a profesores y futuros profesionales en la utilización de muestras auténticas de lengua y cultura en sus clases, utilizando materiales accesibles encontrados en Internet.

Palabras-clave: literatura, arte, contenidos básicos, clases de español.

\section{How to integrate Art and Literature to Spanish classes}

\begin{abstract}
Using Art and Literature in Spanish classes can be a way to learn about the culture and the history of the target language. Besides, it can facilitate the process of learning and the integration of basic contents that are found in didactic books. In this paper, we suggest some ways of using Art and Literature in SFL (Spanish as Foreign Language) classes. By this, we aimed at helping in-service and pre-service teachers to use authentic samples of language and culture in their classes, by utilizing materials that can be found in Internet.
\end{abstract}

Keywords: Literature, Art, basic contents, Spanish classes.

El desarrollo de nuevas prácticas y la elaboración de materiales para el aula de lengua extranjera reflejan, según Richards y Rodgers (2001), el compromiso para encontrar maneras más eficaces de enseñar lengua extranjera. Elaborar programas y garantizar formas más eficientes de enseñar amplía las opciones metodológicas para elegir métodos y materiales de acuerdo con las necesidades de alumnos y profesores. Sin embargo, siguen los autores, los métodos siempre están basados en diferentes puntos de vista, algunos materiales didácticos son difíciles de conseguir y frecuentemente el profesor no encuentra teoría general para basar los contenidos durante las prácticas cotidianas en el aula. De esta manera, algunos temas que involucran la historia, el arte y la literatura normalmente no son utilizados por los profesores como muestras de lengua y cultura por la falta de materiales y de metodologías para su uso en clase.

La experiencia como profesoras de español lengua extranjera (E/LE), en la enseñanza Fundamental y Media de escuelas públicas y el contacto con los alumnos de prácticas del curso de Letras de la Universidad Federal de Goiás, mostró que este tema es uno de los 
motivos de preocupación entre los profesionales con la realidad de las escuelas públicas en Brasil, que no adoptan un manual didáctico para impartir clases de E/LE y dejan a los profesores la tarea de seleccionar y producir lo que utilizarán.

De esta situación surgen preguntas como: ¿Dónde encontrar material? ¿Qué recursos debo utilizar? ¿Qué, cómo y en qué momento abordar los aspectos culturales? ¿Cómo utilizar el arte y la literatura? y si es posible adaptarlos a los contenidos obligatorios exigidos en las clases regulares de español.

Esa fue la motivación que nos llevó a proponer un taller y presentarlo en el III Encontro de Professores de Língua Estrangeira organizado por el Centro de Ensino e Pesquisa Aplicada à Educação (Cepae/UFG) en abril de 2008, que pretendía reunir profesores de la red municipal y estadual de Goiás. El principal objetivo era el de presentarle a los profesores ideas prácticas utilizando muestras auténticas de arte y literatura como un recurso didáctico, adaptándolas a los contenidos básicos de la enseñanza de la lengua meta. Y por fin, presentarles algunos materiales que pueden ser encontrados fácilmente en sitios de Internet.

¿Y porqué utilizar el arte y la literatura y no otras muestras de cultura? Actualmente se reconoce la importancia de enseñar los aspectos culturales en las clases de E/LE a partir de una perspectiva intercultural dónde el alumno conozca la cultura de la lengua meta y reconozca la suya, dónde se pueda propiciar un ambiente favorable a una convivencia de integración ante la diversidad cultural que se presenta y dónde se pueda trabajar la cultura de una sociedad de manera más amplia conociendo sus normas sociales, sus ritos y rutinas que, según Coto y Valderrama (2006), forman parte de la convivencia diaria de una colectividad y ayudan a evitar malentendidos.

La intención era desmitificar la idea de que el profesor necesita conocer profundamente este tema, a punto de inhibirlo y preferir no utilizar estos contenidos en su clase, caso no sea un experto en el asunto arte y literatura.

Para este taller se utilizaron muestras auténticas que forman parte de la llamada cultura con $C$ mayúscula y que para Coto y Valderrama (2006) se trata de algo más amplio y universal como obras inmortales, la música, la literatura, el folclore, la arquitectura, los avances científicos, tecnológicos y acontecimientos históricos de un país o civilización que pueden ser aprendidos o estudiados. Por mucho tiempo enseñar la cultura con $C$ mayúscula fue una práctica común pero con finalidad en si misma, o sea, según Soler-Espiauba (2006) lo importante era adquirir la gramática para traducir los clásicos y no importaba hablar el idioma o conocer los códigos de comportamiento de una sociedad. La cultura legitimada o cultura 
con $C$ mayúscula dejó de ser, según Miquel (2005), esencial en las clases por ser compartida por una minoría.

Lo que se pretende con este taller es de cierta forma rescatar la utilización de la cultura con $C$ mayúscula en las clases de E/LE sin de manera ninguna despreciar los demás contenidos socioculturales. Si de alguna forma nos interesa enseñarla o es necesario a la formación de nuestros alumnos, entonces debe tratarse aunque de manera más superficial, sin que ultrapase lo que el alumno quiera aprender ni tampoco lo que el profesor sea capaz de explicar. Por ese motivo la propuesta fue presentar muestras de cultura con $C$ mayúscula que sirvan como herramienta de enseñanza entre un sinnúmero de recursos didácticos disponibles en Internet para transmitir contenidos básicos de gramática y vocabulario. Seguimos explicando como desarrollar de forma práctica algunas clases utilizando esta propuesta.

\section{EL DESARROLLO}

Según Gargallo (1999) los materiales didácticos para lenguas extranjeras son elaborados por profesionales que cuentan con un conocimiento interdisciplinario en Lingüística, Pragmática, Sociolingüística, metodología entre otros y pueden presentarse en módulos o unidades atendiendo a criterios temáticos o funcionales. Normalmente contienen elementos como muestras de lengua orales o escritas, conceptualizaciones que se refieren a los contenidos gramaticales, funcionales, léxico, fonético-fonológico y ortográfico y por último actividades para practicar los contenidos desarrollados en esa unidad y coherentes con la orientación metodológica subyacente.

El arte y la literatura constituyen un recurso didáctico que puede motivar el interés y auxiliar la comunicación de los alumnos según los distintos temas programados para el curso de E/LE.

Así, el primer paso fue reunir diversos manuales didácticos, analizarlos y seleccionar los contenidos que se repetían en las unidades de cada manual, apenas como referencia, para no llevarle al profesor algún tema que esté fuera de su realidad. Los contenidos analizados fueron divididos en unidades temáticas, como "La familia", "Lugares y localización", "Viviendas", "Los alimentos" y "Descripciones físicas". Los temas que proponemos desarrollar con alumnos son los que comúnmente se trabajan en un nivel inicial e intermediario y los que juzgamos ser el nivel máximo alcanzado para la enseñanza fundamental y media de escuelas públicas en nuestro país. 
Cada unidad temática empezó con una pregunta que está relacionada con la(s) obra(s) de arte o de literatura que servirán de herramienta para desarrollarla. El objetivo es llevar el alumno a buscar y demostrar sus conocimientos implícitos sobre el tema y que pueda relacionarlo con su cotidiano, dónde el profesor, si quiere y el tiempo permite, pueda ampliar las informaciones y trabajar de forma interdisciplinaria con otras asignaturas. A seguir presentaremos cada unidad y las orientaciones para su desarrollo, así como la propuesta de algunos ejercicios que desenvuelvan las cuatro habilidades, expresión oral y escrita, comprensión auditiva y lectora.

\section{$\underline{\text { Unidad temática - La Familia }}$}

¿Qué sabes de Pablo Picasso?

1. Se utiliza la obra "Las Meninas" (Figura 1) de Diego Velázquez, "Retirantes" (Figura 2) de Cândido Portinari y "Miserables Frente al Mar" (Figura 3) de Pablo Picasso, para demostrar las semejanzas y diferencias entre las familias de diferentes épocas y lugares;

2. Se puede explotar los colores de las pinturas originales y la percepción de los alumnos sobre las obras;

3. El profesor puede trabajar la biografía de uno de los artistas como pretexto para introducir el pretérito imperfecto e indefinido;

4. Y, por fin, pedir a los alumnos que cuenten oralmente o por escrito sobre sus vidas.

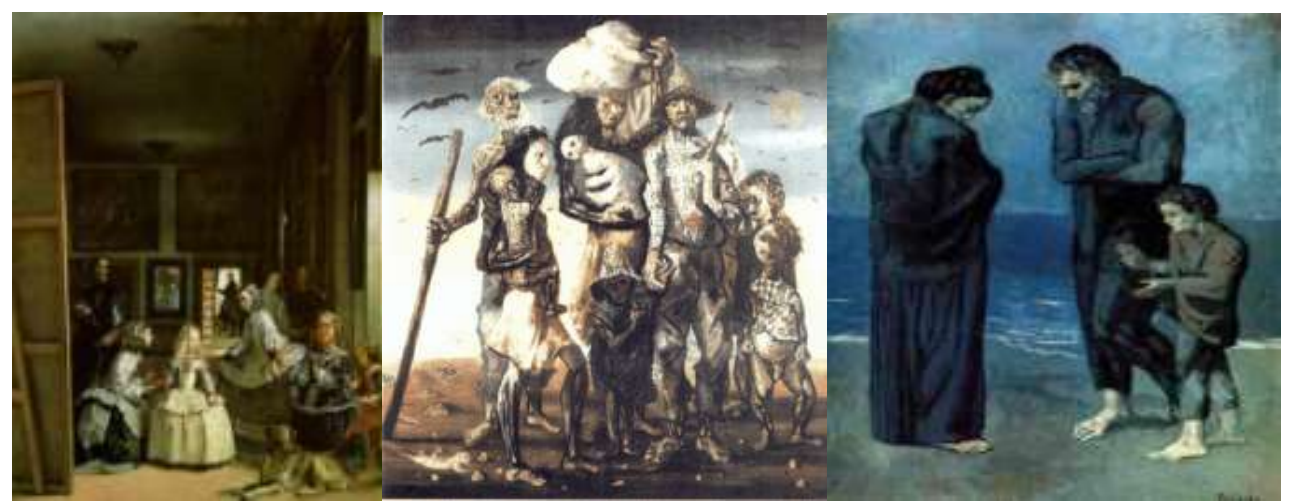

Figura 1 - Las Meninas

Figura 2 - Retirantes

Figura 3 - Miserables frente al Mar

Todas las figuras que se presentan en este trabajo fueron retiradas de sitios en la red mundial de computadores (WEB) así como informaciones sobre los artistas y grabadas en un CD o en láminas para proyección, dependerá de la accesibilidad que tiene el profesor, en su local de trabajo, a la Internet y a los equipamientos (ver referencias). 
Para esta unidad temática se sugiere al profesor que sea realizada en dos clases de 45 minutos, para que tenga tiempo de comentar, aunque de forma sutil, la biografía de los artistas y la época en que fueron pintados estos cuadros. También para que los alumnos puedan explotar características como riqueza, pobreza, hambre, frío, etc., y el profesor introduzca el vocabulario de la familia.

Esta es una actividad que permite un trabajo interdisciplinario con profesores de Artes plásticas o de Historia que mucho tendrán a agregar con informaciones de estos períodos. Así para terminar el tema, en una segunda clase, se trabaja la vida familiar de los alumnos practicando oralmente el vocabulario aprendido e introduciendo el pretérito imperfecto e indefinido con ejercicios orales o escritos.

\section{Unidad temática - Lugares y localización}

¿Dónde queda?

1. Se puede presentar, a nuestros alumnos, obras de Antonio Gaudí como "La Casa Batló" (Figura 4), "La Sagrada Familia" (Figura 5) y con ellas introducir la temática propuesta, a partir de las preguntas ¿dónde queda? ¿dónde está?;

2. Se puede seguir utilizando figuras de otros lugares y preguntando: ¿dónde queda? Machu Picchu, las islas Galápagos, el Cristo Redentor, la Casa Rosada, el Parque Güell, etc. Y de esa forma ampliar los conocimientos;

3. Después se pasa a preguntarles sobre localizaciones de establecimientos que conozcan en su propia ciudad, como un gran almacén, un campo de fútbol, un parque, un hospital, un cine y otros más que el profesor sugiera.

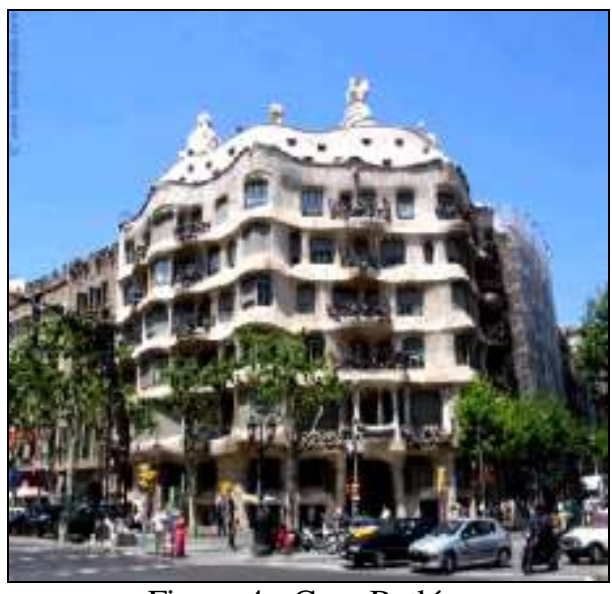

Figura 4 - Casa Batló

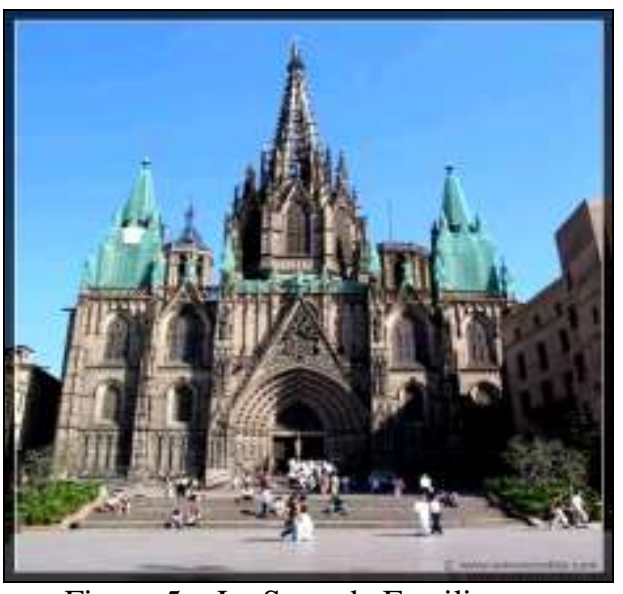

Figura 5 - La Sagrada Familia 
En esta unidad se pretende utilizar las formas arquitectónicas tan sólo como pretexto para introducir el tema y como forma de llamar la atención. Se parte de algo más amplio, recordando nombres de lugares en diversos países y se llega a una realidad más próxima que puede ser la ciudad en que vivimos. De esa forma se trabajan los contenidos básicos sobre localización de lugares, establecimientos, personas y las preposiciones de lugar.

Nada impide que el profesor haga comentarios sobre las figuras que utilizó y amplíe el conocimiento y, dependiendo del interés de los alumnos, proponga una investigación en grupo más profundizada sobre otros lugares.

\section{$\underline{\text { Unidad temática - Los alimentos }}$}

¿Sabes cocinar? ¿Dime qué?

1. La propuesta es preguntarles a los alumnos qué saben cocinar y si hacen esa actividad en sus casas, si les gusta o no, quién cocina en su casa, si hay comidas que le provocan algún sentimiento, le recuerdan algún lugar o momento y otras preguntas relacionadas al tema;

2. Después presentarles pequeños fragmentos, de la obra Como Agua para Chocolate de Laura Esquivel (2008) y pedirles que lean e identifiquen palabras que indican comidas o ingredientes. Aquí se presentan los fragmentos con las posibilidades que el alumno tiene que encontrar. Ya el profesor debe pasárselos sin subrayar.

a) La cebolla tiene que estar finamente picada. Les sugiero ponerse un pequeño trozo de cebolla en la mollera con el fin de evitar el molesto lagrimeo que se produce cuando uno la está cortando. (p. 11)

b) - ¡Este es un placer de los dioses!

Mamá Elena, aunque reconocía que se trataba de un guiso verdaderamente exquisito, molesta por el comentario dijo:

- Tiene demasiada sal. (p. 49)

c) La primera operación es tostar el cacao. Para hacerlo es conveniente utilizar una charola de hojalata en vez del comal, pues el aceite que se desprende de los granos se pierde entre los poros del comal. (p. 143)

d) Tal parecía que por un extraño fenómeno de alquimia su ser se había disuelto en la salsa de las rosas, en el cuerpo de las codornices, en el vino y en cada uno de los olores de la comida. (p. 50) 
3. En un tercer momento se aclaran dudas de vocabulario y se comenta la obra, su autora, los personajes, la historia, las características relacionadas al "Realismo Mágico" sin profundizarse demasiado. Sería muy interesante ver partes de la película (de mismo título) que se refieran a los fragmentos mencionados y las consecuencias que esas comidas provocaban a quien las prueba;

4. Al final se propone ampliar esta clase hablando sobre el "maíz", principal alimento de las Américas y su origen y que los profesores elaboren un ejercicio con recetas de alimentos como en el ejemplo que presentamos para que los alumnos contesten con informaciones de modo de preparo e ingredientes. Se pueden utilizar comidas como el "Taco Mexicano", "la Arepa", "la Cachapa", "La polenta" y otras.

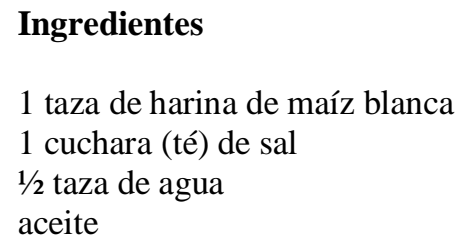
en un recipiente. Agregue la de la sal y el amase con las manos hasta obtener una mezcla suave que se pegue (en las manos). Con la mezcla forme pequeñas pelotitas y achátelas. Caliente la chapa o el horno y coloque las doradas y rellénelas con si prefiere

\section{AREPAS ASADAS}

Esta actividad tiene como objetivo principal introducir el vocabulario de alimentos y llevarle al alumno otras curiosidades de la culinaria hispanoamericana. Con esta obra también podemos desarrollar contenidos gramaticales como la utilización del imperativo y del verbo gustar, así como nociones de cantidad, vocabulario de utensilios de cocina y otros relacionados con la unidad temática estudiada. Para conocer las recetas se puede recurrir a la Internet e incluso grabar vídeos cortos para presentar a los alumnos, sobre la elaboración de cada comida.

\section{$\underline{\text { Unidad temática - Viviendas }}$}


1. Cada alumno puede hacer un pequeño comentario de su casa, si es grande o pequeña, si tiene muchas habitaciones, qué parte de la casa le gusta más, si existen reglas de convivencia y cuáles son, si son cumplidas o no. Es importante escribir en la pizarra todas las palabras nuevas que vayan surgiendo sobre el tema;

2. En un segundo paso se propone la lectura de un fragmento de la obra El cuarto de atrás de Carmen Martín Gaite encontrado en Lorenzo, Hermida y Pino (2006), con el objetivo de ampliar el vocabulario que se refiere a las partes de la casa;

(...) Mi casa de Salamanca tenía dos pasillos paralelos, el de delante y el de atrás, que se comunicaban por otro pequeñito y oscuro, en ése no había cuartos, lo llamábamos el trazo de la hache. Las habitaciones del primer pasillo daban a la Plaza de los Bandos, las del otro lado, a un patio abierto donde estaban los lavaderos de la casa, y eran la cocina, la carbonera, el cuarto de las criadas, el baño y el cuarto de atrás. Era muy grande y en él reinaban el desorden y la libertad, se permitía cantar a voz en cuello, cambiar el sentido de los muebles, saltar encima de un sofá desvencijado y con los muelles rotos al que llamábamos de el pobre sofá, tumbarse en la alfombra, mancharla de tinta, era un reino donde nada estaba prohibido. [...] (p. 126)

3. Como actividad práctica los alumnos deben, según las informaciones del texto y su propia percepción, intentar dibujarla en una hoja fornecida por la profesora. Se sigue, mostrando el dibujo de cada alumno a los demás compañeros para que perciban las posibles diferencias entre ellos;

4. Por último leer juntos el texto aclarando dudas y preguntándoles qué piensan de la casa y de la familia que vivía en ella. ¿Es parecida con la tuya?

Aquí la idea es trabajar con la percepción descriptiva de ambientes de la casa, hacer referencia al hogar de los alumnos y comparándolo con lo mencionado en el fragmento de la obra. Como temas gramaticales se pueden explotar los comparativos, preposiciones, verbos en pasado, además del vocabulario relacionado con la casa.

\section{Unidad temática - Descripciones físicas}

¿Qué te parecen estas figuras?

1. Al mostrar las pinturas de Pablo Picasso "Auto-retrato" (Figura 6), (Figura 7) preguntarles como era antes y después, ¿por qué piensan que está tan diferente? o ¿qué en 
él se ve diferente? En este momento se aprovecha para ampliar el vocabulario y trabajar los diferentes aspectos físicos como, tiene pelo negro, ahora es calvo o pelado, era más joven, ahora está más viejo...

2. También se puede utilizar "Les Demoiselles D’Avigñon" (Figura 8) y "Cabeza de Mujer Llorando" (Figura 9) con el mismo propósito, además se pueden explotar los aspectos psicológicos y de personalidad como ejemplo ¿todas las mujeres te parecen iguales? (Figura 8), ¿percibes que llora? (Figura 9) ¿por qué?

3. Con la obra "Cabeza de Mujer Llorando" (Figura 9) se pueden trabajar aspectos históricoculturales como el período en que esta obra fue concebida, juntamente con "Guernica", una de sus obras más famosas, inspirada por la trágica guerra civil española en 1937.

4. Como actividad proponemos que los alumnos construyan, utilizando revistas, su propio personaje y lo describan a sus compañeros.

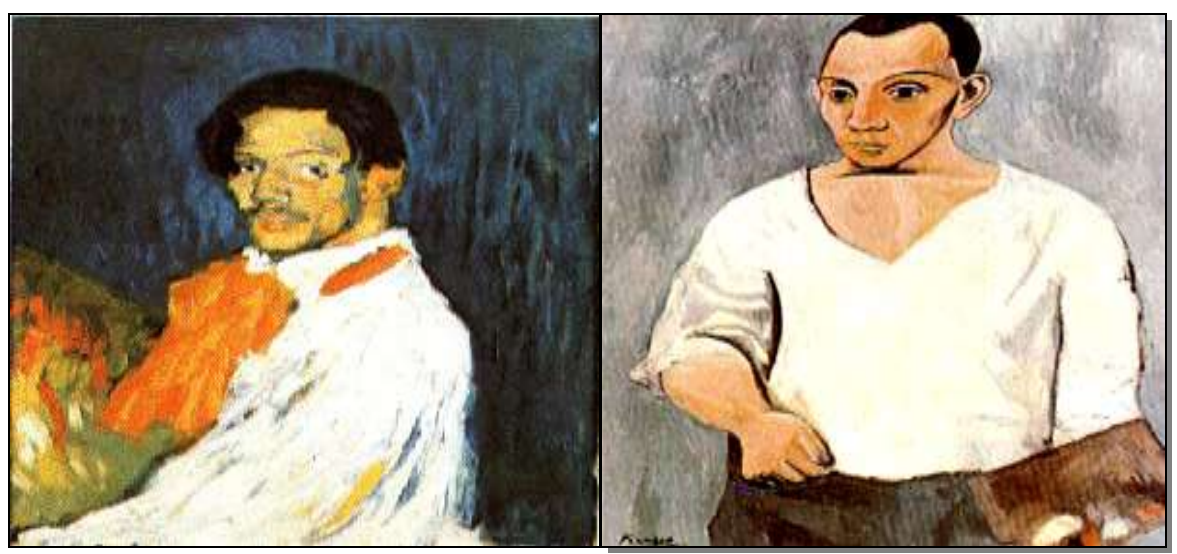

Figura 6 - Auto-retrato

Figura 7 - Auto-retrato

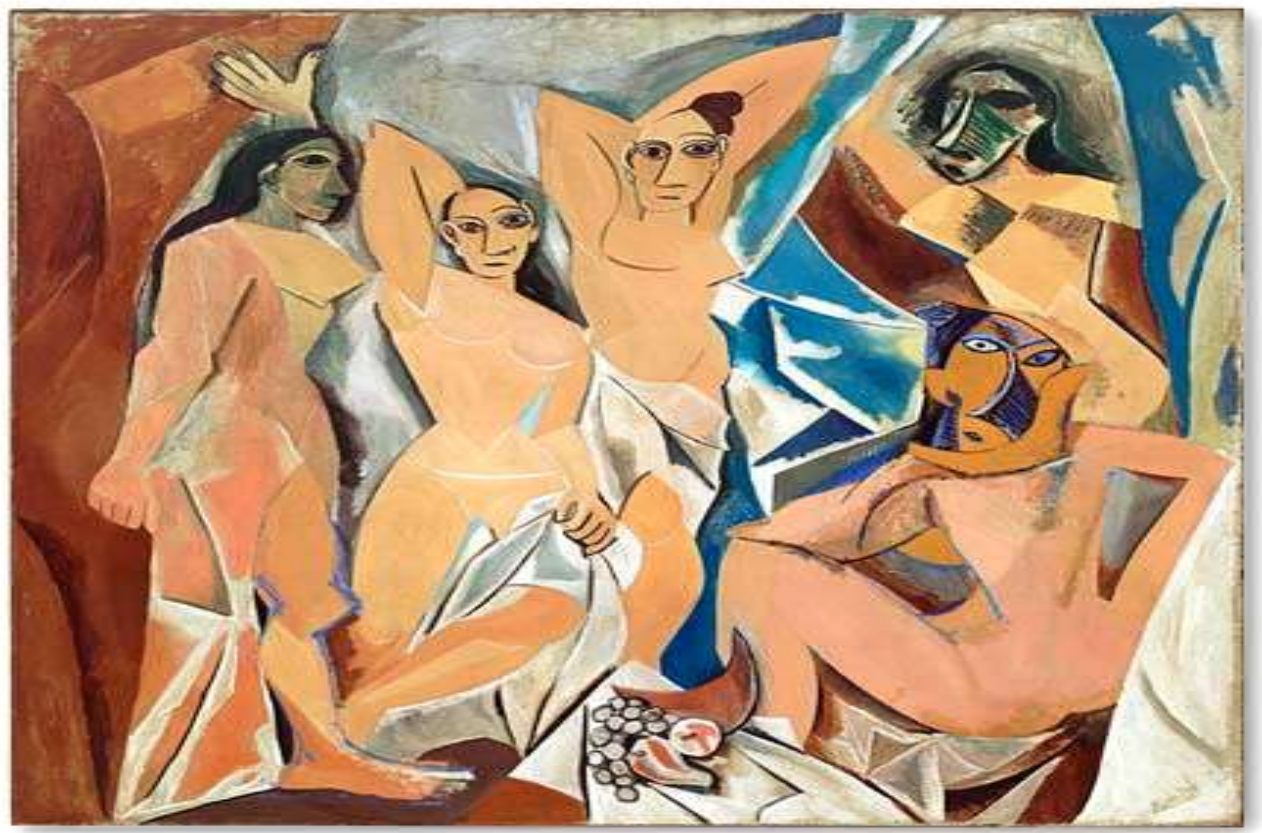

Figura 8 - Les Demoiselles D’ Avigñon 


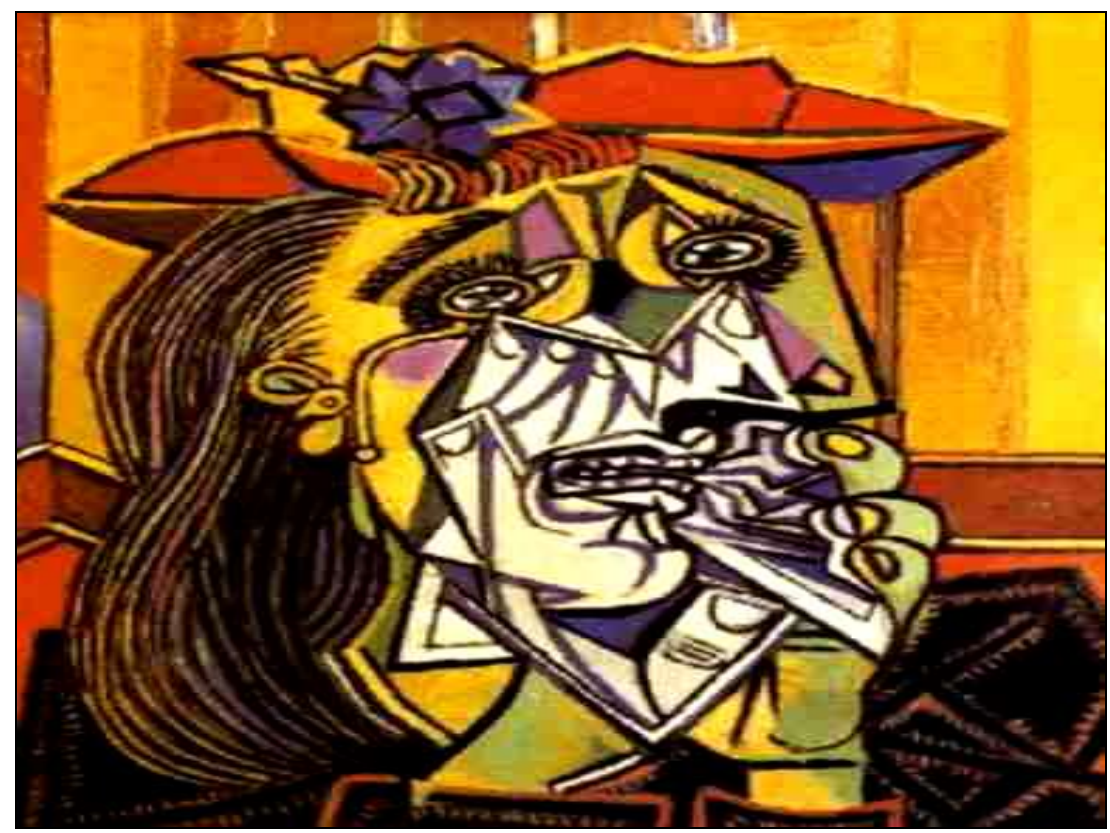

Figura 9 - Cabeza de Mujer Llorando

Esta unidad tiene el propósito de llevar al alumno obras de un artista muy renombrado como Pablo Picasso, que le llamen la atención por sus características estéticas, además de utilizarlas como pretexto para trabajar los aspectos físicos y el vocabulario que se relaciona con el tema. Este también es un buen momento para hablar sobre algunas características del cubismo si los alumnos presentan interés.

Algunas de las informaciones de esta unidad temática se pueden presentar en forma de un pequeño texto y para fijar algún contenido gramatical como los pretéritos.

\section{OBSERVACIONES FINALES}

No era interés de este taller enseñar el profesor a impartir clases de español, mucho menos de arte o literatura, sino que supiera agregarle a sus clases y a los contenidos habituales más informaciones, utilizando como herramienta las obras de arte y de la literatura española e hispanoamericana.

Así como elegimos obras de Picasso, podrían haber sido utilizadas obras de otros artistas, queda a criterio del profesor. Les aconsejamos a los profesores que investiguen otras obras que se adapten mejor a sus clases y a sus alumnos. Las sugerencias de clases que fueron presentadas pueden ser adaptadas a cualquier nivel y propósito de enseñanza del español. Nuestro intento mayor fue el de hacer notar que el arte y la literatura pueden funcionar como 
herramienta y no solamente como tema principal en las clases. Otro reto en este taller fue la utilización de materiales que están disponibles a todos los profesores.

Esperamos que lo que fue discutido aquí pueda servir de base para mejorar la práctica de los profesores y contribuir con la formación de los futuros profesionales.

\section{REFERENCIAS}

BELLO, P. et al. Didáctica de las segundas lenguas: estrategias y recursos básicos. Madrid: Santillana, 1990.

COTO, M. E; VALDERRAMA, Y. F. El componente cultural en la clase de E/LE. Madrid: Edelsa, 2006.

ESQUIVEL, L. Como Agua para Chocolate. 11ªed. Buenos Aires: Debolsillo, 2008.

FERNÁNDEZ, M.; RODRÍGUEZ, C. La enseñanza de la cultura en la clase de español de los negocios. Madrid: Arco Libros, S.L., 2005.

GARGALLO, I. S. Lingüística aplicada a la enseñanza -aprendizaje del español como lengua extranjera. Madrid: Arco Libros, S.L., 1999.

LORENZO, B. R.; HERMIDA, M. F.; PINO, A. N. G. Curso de Literatura: español lengua extranjera. Madrid: Edelsa, 2006.

MAINARDI, B. N.; GASPARINI, F. P. Puentes. Catorce Puntos clave para que los brasileños optimicen su español. São Paulo: Special Book Services Livraria, 2000.

MIQUEL, L. La subcompetencia sociocultural. In: LOBATO, J. S.; GARGALLO, I. S. Vademécum para la formación de profesores: enseñar español como segunda lengua (L2) / lengua extranjera (LE). Madrid: SGEL S.A., 2005.

RICHARDS, J. C.; RODGERS, T. S. Enfoques y métodos en la enseñanza de idiomas. Traducción de José M. Castillo. Madrid: Cambridge University Press, 2001.

SOLER-ESPIAUBA, D. Contenidos culturales en la enseñanza del español como 2/L. Madrid: Arco Libros, 2006.

TUR, J. R. T. (coord.). Coleção Gênios da Arte: Picasso. Tradução Mathias de Abreu Lima Filho. São Paulo: Girassol/Madrid: Susaeta Ediciones, 2007.

www.biografiasyvidas.com >>>acceso en 06 de marzo de 2008.

www.videoposters.es >>>acceso en 06 de marzo de 2008.

www.steimbaugh.com/microsite/arte-de-espana/picasso.html >>>acceso en 09 de marzo de 2008 . 
www.steimbaugh.com/microsite/arte-de-espana/velazquez.html >>>acceso en 09 de marzo de 2008.

www.pt.wikipedia.org/wiki/candido-portinari >>>acceso en 20 de marzo de 2008.

www.es.wikipedia.org/wiki/pablo-picasso >>>acceso en 20 de marzo de 2008.

www.tierra.free-people.net/artes/pintura-pablo-picasso.php >>>acceso en 01 de abril de 2008.

www.tierra.free-people.net/artes/pintura-diego-velazquez.php >>>acceso en 01 de abril de 2008.

www.tierra.free-people.net/artes/arquitectura-antonio-gaudi.php >>>acceso en 01 de abril de 2008.

www.tierra.free-people.net/artes/literatura-laura-esquivel.php > > acceso en 01 de abril de 2008.

www.tierra.free-people.net/artes/literatura-carmen-gaite.php >>>acceso en 01 de abril de 2008. 